\title{
A Rule Based Decision Support System for Aiding Vitamin D Deficiency Management
}

\author{
Muthuraman Thangaraj' and Sankaran Gnanambal ${ }^{*}$ \\ ${ }^{1}$ Associate Professor, Department of Computer Science, Madurai Kamaraj University, Madurai, Tamil Nadu, India; \\ thangarajmku@yahoo.com \\ ${ }^{2}$ Research Scholar, School of Computer Science, Bharathiyar University, Coimbatore, Tamil Nadu, India; \\ gnanambal.ambal@yahoo.com
}

\begin{abstract}
The objective of this study is to diagnose vitamin D deficiency for which rule based Decision Support System (DSS) was used. This diagnosis task was achieved through neuro-fuzzy classifier. For this, we constructed an ontology related to food supplements for the management of vitamin D deficiency. The Semantic Web Rule Language (SWRL) is used to create rules corresponding to vitamin D deficiency management. Finally, Java Expert System Shell (JESS) has to be used for reasoning, which provides appropriate food items for vitamin D deficiency management.
\end{abstract}

Keywords: DSS, Vitamin D Management, Ontology, SWRL, JESS.

\section{Introduction}

The dramatic growth of technological development in healthcare systems has to be recorded through a variety of methods ${ }^{1}$. The Decision Support System (DSS) is one of the methods used in healthcare systems. DSS is an interactive application, which is developed to support physicians, and healthcare professionals for decision making $^{2}$. It is one of the major areas in Artificial Intelligence in Medicine. It generates case-specific advice from patient data $^{3}$. DSS uses clinician's knowledge, and then, provides a better analysis of the patient's data. Therefore, DSS provides suggestions about outputs, and the physician retrieves significant information and removes incorrect suggestions ${ }^{4}$.

DSS can support healthcare professionals to analyze the characteristics of patients and produce treatment recommendation, follow-up, and monitoring. Medical domains contain huge amount of complex information, therefore, ontologies are used to reuse that complex information. Ontology is a combined approach of artificial intelligence, and machine learning with sharing and reusability of knowledge ${ }^{5}$. Ontologies are used to construct systems in the field of information retrieval and natural language processing, but it is most useful in the field of healthcare systems to provide personalized treatment. The constructed knowledge base in the ontology can be reused in new systems.

Classification plays a prominent role in medical diagnosis systems ${ }^{6}$. There are many classifiers available today, such as Decision trees, Navie bayes, K-nearest neighbor, Support vector machine, neural networks and so on $^{7,8}$. In the last few years, research has been carried out to combine neural networks and fuzzy systems to construct Neuro-Fuzzy Classifiers (NEFCLASS). The NEFCLASS is used to identify the correct class or category of a given input population'. Neuro-fuzzy method is applied to construct a fuzzy system from data through learning methodthat is stipulated by learning procedures used in neural networks ${ }^{10}$.

In this study, we present a framework for aiding vitamin D deficiency. The framework system consists

*Author for correspondence: 
of Decision making environment, and it generates classification rules using Neuro-fuzzy classifier. From the generated rules, the system provides the decision making with respect to current status of the patient such as age and laboratory test results. Based on the result of decision making environment, the system activates the SWRL rule that, in turn, provides the treatment flow.

Vitamins are needed for normal body function, and our body can't produce them by itself. But vitamin $\mathrm{D}$ is something different. That is our body can produce vitamin D with sunlight exposure mostly, and a little from a few food supplements. Vitamin D is a hormone and it maintains the dietary calcium and phosphate level in the blood as normal. Therefore, vitamin D plays a vital role for maintaining bone strength ${ }^{11}$. Deficiency in vitamin D and/or less calcium intake could cause bone mineralization. This deficiency arises due to inadequate sunlight exposure, and insufficient dietary calcium intake. According to Indian Council of Medical Research reports, there is a widespread vitamin D deficiency in Asian Indians ${ }^{12}$. The studies conducted in school going children by national Institute of Nutrition, Hyderabad show that there is a vital contribution on vitamin $\mathrm{D}$ for determining their height and bone health ${ }^{13}$. For maintaining vitamin $\mathrm{D}$ and calcium intake is not an easy task. In this study, we propose Food Ontology for the management of Vitamin D Deficiency (FO-VDD).

\section{Materials and Methods}

NapatSuksom et al., ${ }^{14}$ presents a Personalized Food Recommender System (PFRA) that supports the users in daily diet recommendations, based on the nutrition guidelines. This paper provides food ontology and recommender system through the knowledge-based framework. The developed food and nutrition ontology is combined with rule-based knowledge for providing food recommendations, corresponding to user's nutrition requirements, and food preferences. But the implementation of the system has not been evaluated in a scientific way.

Papageorgiou ${ }^{15}$ constructs a framework, which is based on Fuzzy Cognitive Map (FCM) and fuzzy rule extraction techniques. This framework is a combined approach of fuzzy logic and neural networks, which makes the system as more knowledgeable. The main drawback of the system framework is weak due to lack of information support.

Alexandrov et al., ${ }^{16}$ present the Sempath (Semantic Clinical Pathway) Software environment that provides a personalization of the treatment plans for a single patient. The term personalization requires the continuous reconfiguration and adaptation of the treatment schemes. During the execution time of Sempath, the system reasons over the rules and provides next steps of the treatment. But the implementation of the pathways has not been tested in a scientific way.

In this study, we present a fully automated healthcare model for managing vitamin D deficiency, which provides vitamin $\mathrm{D}$ deficiency diagnosis and treatment flow. The architecture of the proposed model is given in Figure 1 and comprises three environments:

a) NEFCLASS based decision making environment.

b) SWRL Rule construction and execution environment.

c) Ontology construction.

These three environments are described in detail in the following sections.

\subsection{NEFCLASS based Decision Making Environment}

This environment handles the generation of classification rules using neuro-fuzzy classifiers from a dataset. The NEFCLASS includes three layers, first for input, second for rules, and third for output. The input layer processes input data. The activation function of input neuron is generally an identity function, and it can do preprocessing. The rule layer is used to represent the fuzzy rules. The activation function of neurons in rule layer is calculated through the weight of input neuron and rule neuron. The output layer is used to predict the class. The activation function of output neuron is calculated via weight between rule neuron and output neuron. The neuron with the highest activation function is predicted as a result of classification. The NEFCLASS generates the fuzzy rules as a result of classification. The generated classification rules are stored into rule repository. The rule repository is developed by the use of Business Rule Management System (BRMS). This study uses open rules $\mathrm{BRMS}^{17}$ as a tool to construct the rule repository and the rule engine. At the time of execution, the rule engine collects all matched rules corresponding to patient's current status. A rule engine executes the matched rules, and it produces a diagnosis result such as vitamin D deficiency, insufficiency, healthy and toxicity. The pseudo code of the NEFCLASS is presented in Table 1. 
Table 1. Pseudo code of the NEFCLASS algorithm

Pseudo code of the NEFCLASS:

Step 1: Input the training data and find all supported antecedents $\mathrm{K}_{\mathrm{i}}$ (where $\left.\mathrm{i}=1,2, \ldots \mathrm{n}\right)$ and create initial antecedents set.

Step 2: For each antecedent's $\mathrm{K}_{\mathrm{i}}$,

Create a rule $R_{j}$ for each class (where $j=1,2, \ldots m$ ), construct a initial rule base, that has $\mathrm{R} * \mathrm{~K}$ rules

Step 3: For each rule $\mathrm{R}_{\mathrm{j}}$ evaluate the frequency of variables and select the rules that has same antecedents but different consequents.

Step 4: Resolve the inconsistencies that arise in rules Step 5: Construct a final rule base.

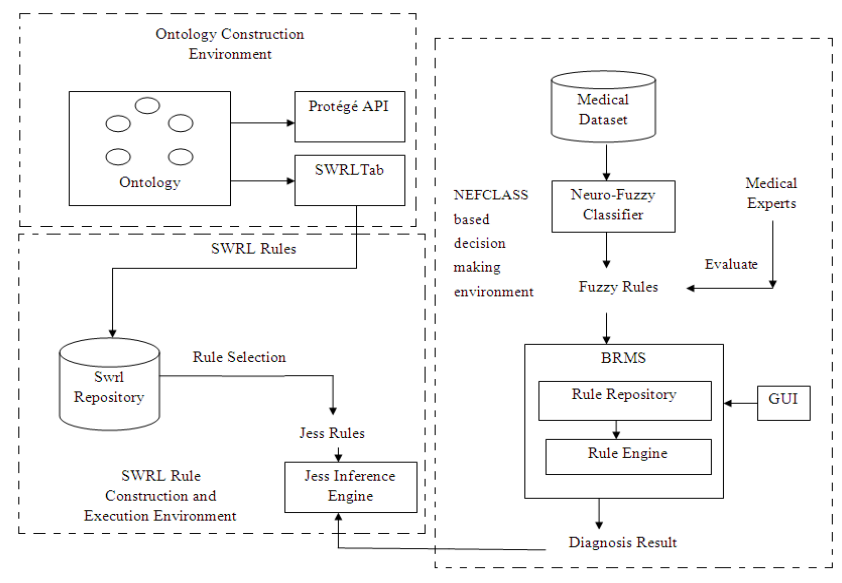

Figure 1. Technical architecture.

Vitamin D deficiency is diagnosed by the 25-hydroxyvitamin $\mathrm{D}(25-\mathrm{OH})$ test $^{18}$. Some of the generated rules are presented below,

\section{If 25-OH is low then deficiency}

If 25-OH is medium then insufficient

If 25-OH is high then healthy

If $25-\mathrm{OH}$ is very high then vitamin-D toxicity

\subsection{SWRL Rule Construction and Execution Environment}

This environment manages with the creation SWRL rules, and the rules are executed via inference engine. The classification rules derived from decision making environment are used to derive diagnosis of vitamin D level for an individual patient. Based on the decision making, the corresponding SWRL rules are selected from SWRL rule repository. The SWRL rule repository contains all the constructed rules related to vitamin D deficiency management. After the selection of matched rules, the rules are executed by JESS inference engine ${ }^{19}$. The JESS produces the result and the new derived facts are updated into the ontology. Three example SWRL rules are presented below.

RULE 1: If the Patient is an adult, and the diagnostic message is deficiency, the rule 1 produces the information about the drug califerol and duration of treatment.

Patientinfo (? p) ^has age (? p, Adult)^Diagnosismsg (? d) ^has-diagnosis (? d, deficiency) ^has Treatment $(?$ p,t) $\wedge$ has Drug (?t,?drug) $\wedge$ Same As (?drug,calciferol) $\wedge$ has-IU (?drug,?iu)^temporal: has StartTime (?t, ?start) $\wedge$ temporal:hasFinishTime (?t, ?finish) $\rightarrow$ sqwrl:select (?p, ?drug, ?iu, ?start, ?finish)

Rule 2: If the Patient is an adult, and the diagnostic message is insufficiency, the rule 3 produces the food items with more vitamin D consumption.

Patientinfo (?p) ^ has age (?p, Adult)^Diagnosismsg (?d) $\wedge$ has-diagnosis (?d, insuffiency) $\wedge$ food-categories (?f) $\wedge$ hasvd-value (?f, ?v) $\rightarrow$ sqwrl:select $(? f, ? v) \wedge$ sqwrl:equal (?v, More)

RULE 3: If the Patient's diagnostic message is healthy, and the selected food group is cereals, the rule 3 produces the cereal items with their vitamin $\mathrm{D}$ consumption value.

Patientinfo (?p) ^Diagnosismsg (?d) ^has-diagnosis $(? d$, healthy $) \wedge$ food-categories $(? f) \wedge$ hasselected-group $(? f, ? g) \wedge$ SameAs (?g, Cereals $) \wedge$ hasvd-value $(? g, ? v) \rightarrow$ sqwrl:select $(? p, ? f, ? v)$

\subsection{Ontology Construction Environment}

The proposed ontology FO-VDD is constructed by using protégé ontology editor, an open source ontology tool from Stanford University. The ontology is represented in owl format ${ }^{20}$. Ontology describes the knowledge, which is used for the creation of SWRL rules. We define our ontology in owl by using the protégé ontology editor. SWRL protégé - plug-in is utilized for the creation of SWRL rules. It provides the interaction between SWRL rules and ontology. First with the help of a physician, we analyze the terms and concepts related to diet. Three classes defined in meta-ontology are represented as food categories, diagnostic message, and patient information. One class could have subclasses or may directly include individuals. For example, food categories include six groups of sub classes 
Cereals, Fruits and Juices, Vegetables, Milk-Products, Fat or Oils, and Meats. The diagnosis-message class possesses four categories, such as deficient, insufficient, healthy and toxicity. Figure 2 shows the part of the developed ontology for vitamin $\mathrm{D}$ management by using protégé-Jambalaya plug-in ${ }^{21}$.

The food composition database constructed by Health Finland ${ }^{22}$ is used for the vitamin D consumption of each and every food item. The vitamin D level of each food item has been collected and analyzed completely to construct the ontology for vitamin $\mathrm{D}$ maintenance. Table 2 shows some of the food items with their vitamin D level per 100 gram.

\section{Performance Analysis}

The proposed system for vitamin D maintenance is implemented with Java 1.6.0, protégé 3.6 and weka 3.7. The experimental environment is constructed to decide the efficiency of the system by comparing it with the PFRA and the proposed system vitamin D deficiency management.

In Figure 3, we measure the performance of rule generation related to the classification algorithms of NEFCLASS and Fuzzy Supervised Learning In Quest (SLIQ) based Decision Tree (FS-DT) ${ }^{23}$ for a metabolic syndrome dataset. In order to evaluate the application, and to find the performance, about 100,000 records have

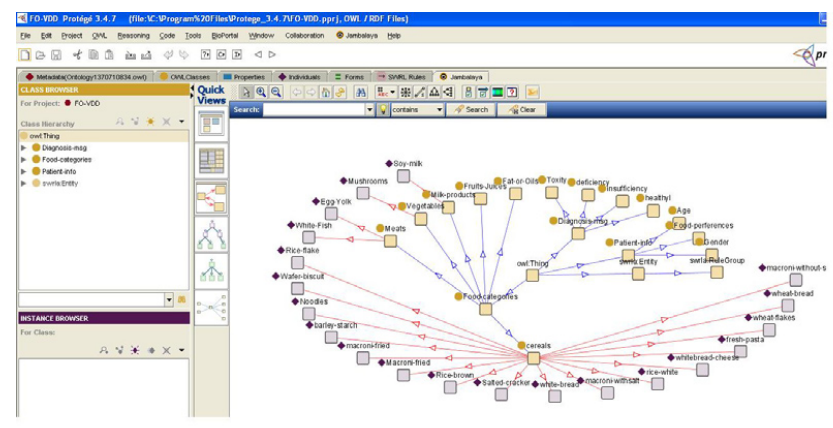

Figure 2. Design of the FO-VDD ontology in protégéJambalaya plug-in.

Table 2. Food items with their vitamin D level

\begin{tabular}{lc}
\hline Item & Vitamin D level (per 100g) \\
\hline White Fish & 24.2 \\
Cod liver oil & 20.0 \\
Mushroom & 15.8 \\
Egg yolk & 6.8 \\
Soy milk & 5.9 \\
\hline
\end{tabular}

been constructed. NEFCLASS algorithm generates more number of rules than FS-DT algorithm.

Figure 4 presents the user's satisfaction level about the diet recommendation of PFRA and FO-VDD. The user's satisfaction level is evaluated by five volunteers i.e., the volunteers make a self-evaluation for his/her recommended diet plan, provided by the system, and then gives a feedback of the system that includes very dissatisfied $(0 \%-30 \%)$, dissatisfied (30\%-60\%), satisfied $(60 \%-80 \%)$ and very satisfied $(80 \%-100 \%)$. This figure shows that the user's satisfaction level of FO-VDD is more effective when compared with PFRA.

Figure 5 shows the satisfaction degree about the diet recommendation of PFRA and FO-VDD. Satisfaction degree is measured by three Domain Experts (DE). The domain experts evaluate recommended diet plan provided by the system, and then give a feedback of the system.

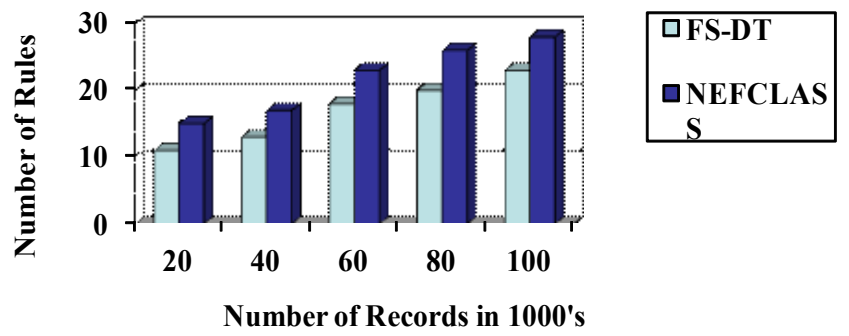

Figure 3. Records vs. rules for vitamin D dataset.

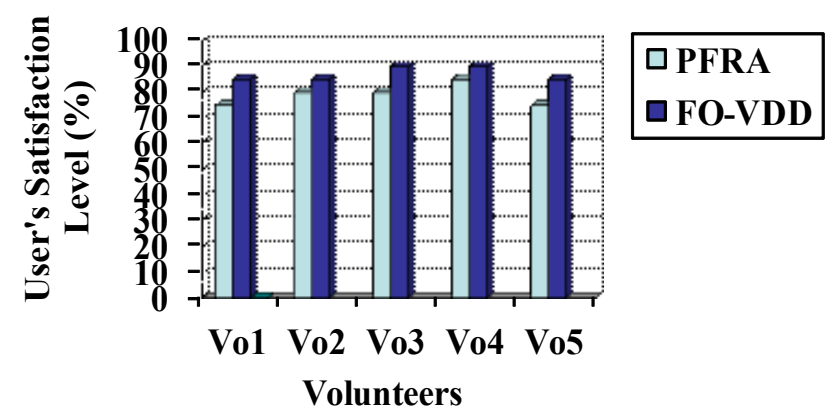

Figure 4. User's satisfaction level evaluated by each volunteer.

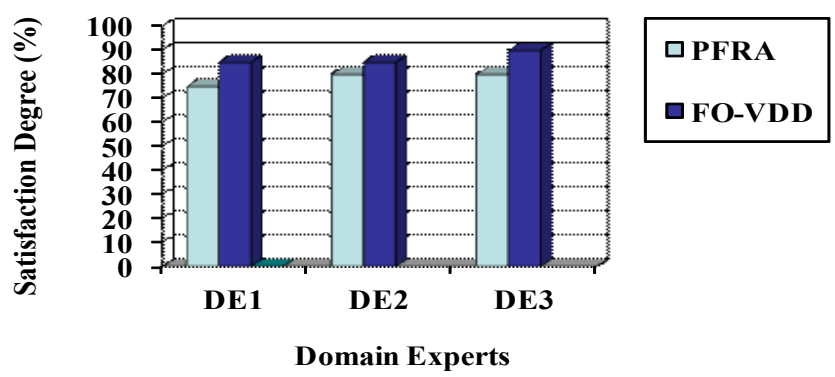

Figure 5. Satisfaction degree evaluated by domain experts. 
The satisfaction degree also includes very dissatisfied (0\%-30\%), dissatisfied (30\%-60\%), satisfied (60\%-80\%) and very satisfied $(80 \%-100 \%)$. This figure shows that the satisfaction degree of FO-VDD is more effective when compared with PFRA.

\section{Conclusion}

The application and the techniques proposed are found to be feasible and user friendly. In this system, an efficient result is achieved, and its application is less time consuming. First, the NEFCLASS algorithms and BRMS are used to provide diagnosis about vitamin D deficiency. Thereafter, based on the diagnosis, the recommendation about diet maintenance is to be provided through ontology, SWRL rules, and JESS. This system provides a supportive interface for training medical students. Vitamin management and maintenance is a complex task in itself, since it is difficult to visit physician or dietician on regular basis. Instead, the proposed automatic food recommendation system for vitamin management acts as a helping hand for people by saving many medical formalities, and also reducing the time of domain experts.

\section{References}

1. Abbasi MM, Kashiyarndi S. Clinical decision support systems: a discussion on different methodologies used in Health Care [Internet]. 2010 Available from: http://www. idt.mdh.se/kurser/ct3340/ht10/FinalPapers/15-abbasi_ kashiyarndi.pdf

2. Chen CC, Chen K, Hsu CY, Li YC. Developing guideline based decision support systems using protégé and jess. Comput Methods Programs Biomed. 2011; 102(3): 288-94.

3. Dhar V, Stein R. Intelligent decision support methods: the science of knowledge work. 4th ed. Upper Saddle River, NJ: Prentice-Hall; 1997.

4. Yao W, Kumar A. CONFlexFlow: Integrating flexible clinical pathways into clinical decision support systems using context and rules. Decis Support Syst. 2012; 55(2):499-515. doi: org/10.1016/j.dss.2012.10.008 [In press]

5. Borst WN. Construction of engineer ontologies $[\mathrm{PhD}$ thesis]. Enschede: University of Twenty; 1977.

6. Anooj PK. Clinical decision support system: risk level prediction of heart disease using weighted fuzzy rules. Journal of King Saud University - Computer and Information Sciences. 2012; 24(1):27-40.

7. Burges C, Scholkopf B. Improving the accuracy and speed of support vector machines. Neural Information Processing Systems. 1997; 9:375-81.
8. Haykin S. Neural networks: a comprehensive foundation. 2nd edition. USA: Prentice Hall; 1999.

9. Nauck DD. Fuzzy data analysis with NEFCLASS. Int J Approx Reason. 2003; 32(2-3):103-30.

10. Gliwa B, Byrski A. Hybrid neuro-fuzzy classifier based on NEFCLASS model. Comp Sci. 2011; 12:115-35.

11. Pearce SHS, Cheetham TD. Diagnosis and management of vitamin D deficiency. Clinical Review, BMJ. 2010; 340 (b5664):142-47.

12. Goswami R, Mishra SK, Kochupillai N. Prevalence \& potential significance of vitamin deficiency in Asian Indians. Indian J Med Research. 2008; 127(3):229-38.

13. Harinarayan CV, Ramalakshmi T, Prasad UV, Sudhakar D. Vitamin D status in Andhra Pradesh: a population based study. Indian J Med Res. 2008; 127(3):211-18.

14. Suksom N, Buranarach M, Thein YM, SupnithiT, Netisopakul P. A knowledge-based framework for development of personalized food recommender system. In: Theeramunkong T, Kunifuji S, Sornlertlamvanich V, Nattee C, editors. The Fifth International Conference on Knowledge, Information and Creativity Support Systems; 2010 Nov 25-27; Chiang Mai: Thailand. 2010. p. 274-277.

15. Papageorgiou EI. A new methodology for decisions in medical informatics using fuzzy cognitive maps based on fuzzy rule-extraction techniques. Appl Soft Comput. 2011 Jan; 11(1):500-13. doi:10.1016/j.asoc.2009.12.010.

16. Alexandrov DA, Skitsas IE, Mentzas GN. A holistic environment for the design and execution self-adaptive clinical pathways. IEEE Trans Inf Technol Biomed. 2011; 15(1):108-18. doi:10.1109/TITB.2010.2074205.

17. OpenRules Inc. OpenRules BRMS [homepage on the Internet]. 2009. [Accessed May 25, 2011]. Available from: www.openrules.com

18. Cannell JJ, Hollis BW, Zasloff M, Heaney RP. Diagnosis and treatment of vitamin D deficiency. Expert Opin Pharmacother. 2008; 9(1):1-12.

19. Friedman-Hill E. Jess the rule engine for the Java ${ }^{\mathrm{TM}}$ platform [Internet]. California : Sandia National Laboratories, Sandia Corporation; 2008 [cited June 2010]. Available from: http://herzberg.ca.sandia.gov/

20. Giri K. Role of ontology in semantic web. DESIDOC Journal of Library and Information Technology (DJLIT). 2011; 31(2):116-20.

21. Stanford University. Jambalaya. [homepage on the Internet]. 2004. Available from: http://protegewiki.stanford.edu/wiki/ Jambalaya_2.6.0

22. National Institute for Health and Welfare, Finland. Fineli-Finnish Food Composition Database. [Internet]. 2010. Available from: http://www.fineli.fi/index. php?lang=en

23. Chandra B, Paul Varghese P. Fuzzy SLIQ decision tree algorithm. IEEE Trans Syst Man Cybern B Cybern. 2008; 38(5):1294-01. doi:10.1109/TSMCB.2008.923529. 